\title{
Electronic shell structure and chemisorption on gold nanoparticles
}

\author{
Larsen, Ask Hjorth; Kleis, Jesper; Thygesen, Kristian Sommer; Nørskov, J. K.; Jacobsen, Karsten Wedel
}

Published in:

Physical Review B Condensed Matter

Link to article, DOI:

10.1103/PhysRevB.84.245429

Publication date:

2011

Document Version

Publisher's PDF, also known as Version of record

Link back to DTU Orbit

Citation (APA):

Larsen, A. H., Kleis, J., Thygesen, K. S., Nørskov, J. K., \& Jacobsen, K. W. (2011). Electronic shell structure and chemisorption on gold nanoparticles. Physical Review B Condensed Matter, 84(24), 245429.

https://doi.org/10.1103/PhysRevB.84.245429

\section{General rights}

Copyright and moral rights for the publications made accessible in the public portal are retained by the authors and/or other copyright owners and it is a condition of accessing publications that users recognise and abide by the legal requirements associated with these rights.

- Users may download and print one copy of any publication from the public portal for the purpose of private study or research.

- You may not further distribute the material or use it for any profit-making activity or commercial gain

- You may freely distribute the URL identifying the publication in the public portal 


\title{
Electronic shell structure and chemisorption on gold nanoparticles
}

\author{
A. H. Larsen, ${ }^{1}$ J. Kleis, ${ }^{1}$ K. S. Thygesen, ${ }^{1}$ J. K. Nørskov, ${ }^{2}$ and K. W. Jacobsen ${ }^{1}$ \\ ${ }^{1}$ Center for Atomic-scale Materials Design, Department of Physics Technical University of Denmark, DK-2800 Kongens Lyngby, Denmark \\ ${ }^{2}$ SUNCAT Center for Interface Science and Catalysis, SLAC National Accelerator Laboratory 2575 Sand Hill Road, \\ Menlo Park, California 94025, USA
}

(Received 3 October 2011; revised manuscript received 22 November 2011; published 14 December 2011)

\begin{abstract}
We use density functional theory (DFT) to investigate the electronic structure and chemical properties of gold nanoparticles. Different structural families of clusters are compared. For up to 60 atoms we optimize structures using DFT-based simulated annealing. Cluster geometries are found to distort considerably, creating large band gaps at the Fermi level. For up to 200 atoms we consider structures generated with a simple EMT potential and clusters based on cuboctahedra and icosahedra. All types of cluster geometry exhibit jelliumlike electronic shell structure. We calculate adsorption energies of several atoms on the cuboctahedral clusters. Adsorption energies are found to vary abruptly at magic numbers. Using a Newns-Anderson model we find that the effect of magic numbers on adsorption energy can be understood from the location of adsorbate-induced states with respect to the cluster Fermi level.
\end{abstract}

\section{INTRODUCTION}

A major theme in advanced materials design today is the possibility to modify and change materials properties through structuring at the nanoscale. The applications can be as diverse as optimizing the size of metal nanoparticles to catalyze certain chemical reactions ${ }^{1}$ or the structuring of surfaces and interfaces for optimal light absorption in photovoltaic devices. ${ }^{2}$ In very broad terms the interesting possibilities arise when the structures reach a scale comparable to the wavelengths of the relevant quantum particles (electrons, plasmons, or photons).

In this work we investigate theoretically the properties of freestanding metal nanoparticles made of gold in particular. The purpose is to improve our understanding of the relationship between cluster size and a range of electronic and chemical properties. Different aspects of this have been investigated in numerous studies. See, for example, the review by Baletto and Ferrando. ${ }^{3}$ What is special here is that we investigate the cluster properties over an-for electronic structure calculationsunusually large size range and for many different cluster structures. The hope is thereby to get a more complete picture of the general trends in the cluster behavior.

For transition metals with partially filled $d$ bands, cohesive energies will be dominated by the effect of the $d$ states. ${ }^{4}$ Because of the short range of the $d$ states, their contribution to the cluster energy is determined mostly by the local arrangement of neighboring atoms. Facet types and local atomic packing can therefore be expected to be particularly important factors in the structures of transition-metal clusters with partially filled $d$ bands.

The effect of the partially occupied $d$ band disappears for noble metals and alkali metals. Instead, the long-range $s$ electrons, which hybridize in a more complex manner, yield the primary contribution to the cluster energy. The optimal structure will not be determined by optimizing the local structure around each atom, but rather by optimizing the global geometric structure to obtain the most desirable electronic structure of the delocalized electron cloud. The result is a much more complicated interplay between electronic and geometric structure.
Small free-standing gold clusters have been theoretically shown to possess very diverse ground-state geometries depending on cluster size. Examples are planar, cagelike, and tubelike structures. ${ }^{5-8}$

The $s$-electron hybridization can be interpreted in terms of a jellium model which regards the whole cluster as a spherical superatom. The $s$ electrons organize into global shells, resulting in electronic "magic numbers" when shells are filled. Magic numbers at 2, 8, 18, 20, 34, 40, 58, . ., have been observed as particularly stable alkali-metal clusters ${ }^{9,10}$ with large band gaps, in agreement with theory. For alkali metals, magic numbers attributed to both electronic and geometric shell structures have been observed for clusters with thousands of atoms, with geometric shells dominating beyond 2000 atoms. ${ }^{11}$ Larger Au clusters are believed to form icosahedra, decahedra, or truncated octahedra depending on size and temperature. ${ }^{12-14}$

In this work we consider several series of clusters based on different generation procedures and structural motifs. We calculate structures of smaller clusters using simulated annealing with density functional theory (DFT) and for larger clusters using effective medium theory ${ }^{15,16}$ (EMT). Using DFT we compare the energy and electronic structure of optimized clusters with the commonly considered regular icosahedral and cuboctahedral structures. For the cuboctahedra we identify trends in reactivity by considering adsorption of different atoms. The geometric similarity of clusters based on cuboctahedra and icosahedra allows us to isolate and study size-dependent effects on chemistry. The price of this simplification is that individual calculations do not represent globally optimal structures. Hence, we focus on trends that are general enough to be significant outside the model systems.

\section{COMPUTATIONAL METHODS}

All electronic structure calculations are performed with the real-space DFT code GPAW ${ }^{17,18}$ using the RPBE ${ }^{19}$ functional for exchange and correlation. GPAW uses the projector-augmented wave (PAW) method ${ }^{20}$ and offers an accurate real-space representation of the Kohn-Sham orbitals along with an efficient 
basis set of localized atomic orbitals. ${ }^{21}$ The calculations presented here are performed with the atomic orbitals using a double- $\zeta$ polarized (DZP) basis set. All calculations on clusters are spin paired and use the scalar-relativistic atomic PAW setups and basis sets supplied with GPAW. The Au setup contains 11 valence electrons.

In our calculations the cluster is centered in a nonperiodic orthorhombic cell with $5.0 \AA$ vacuum along each axis. We use a grid spacing of $0.2 \AA$.

We do not apply any basis set superposition error correction, so the values of adsorption energies are not necessarily accurate. However, in comparing the bonding of an adsorbate to clusters of different sizes, the local structure around the adsorbate is highly similar for all clusters, and the basis set error is consequently roughly the same for all clusters. Therefore, variations in adsorption energies are subject to a much smaller error.

Pulay density mixing ${ }^{22}$ is used to speed up convergence of the self-consistency loop. Electron occupations are smeared by a small Fermi temperature of $0.01 \mathrm{eV}$, which helps speed up convergence.

Structure optimizations are performed using the BFGS algorithm as implemented in the Atomic Simulation Environment ${ }^{23}$ (ASE) and terminate when no force on any atom is larger than $0.075 \mathrm{eV} / \AA ̊$.

\section{CLUSTER GEOMETRY}

Systematic calculation of lowest-energy structures from first principles is computationally very expensive. Previous studies of structures and properties of $\mathrm{Au}$ clusters have therefore usually been limited to a few dozen atoms. ${ }^{8,24-26}$ Here we focus mainly on larger clusters which are quite challenging to systematically optimize and characterize, but which are clearly of interest both from a conceptual point of view and in applications like catalysis. The transition from smaller clusters over larger clusters to bulklike behavior has been studied recently by Kleis et $a .^{27}$ and the results presented here can be seen as a supplement and expansion of this study.

In this work we compare clusters generated by several different procedures. For the smallest clusters we perform simulated annealings using DFT to obtain realistic structures. This is clearly the most realistic and theoretically satisfactory method since the same energy landscape is used to define the cluster geometry or shape as is used to subsequently study the bonding of the cluster and the chemical properties. However, for reasons of computer time this approach cannot be generally applied for larger clusters.

Larger clusters are studied using DFT, but with the structures being determined by simulated annealing with a classical EMT potential. As this EMT potential does not incorporate explicit electronic structure, the structures generated by this method will have no information about potential electronic shell effects but only of atomic shell effects related to atomic packing of the clusters.

We finally construct clusters based on prescribed cuboctahedral and icosahedral shapes. The simplicity of the fcc-based cuboctahedral structures allows us to study adsorption of atoms in a way which preserves the local geometry around the adsorbate for different cluster sizes. This allows us to separate the effect of local geometry from that of the electronic structure of the cluster, which would not generally be possible if the cluster were based on a global minimum search. The comparison of distinct types of structures will help determine how properties of clusters depend on structure versus size.

\section{A. Simulated annealing with DFT}

For the smallest clusters $(N=6-60)$ we calculate realistic geometries using DFT with coarse parameters.

For each size of cluster we perform a rough simulated annealing based on molecular dynamics (MD) to find the optimal structure. We use a Langevin thermostat to regulate the temperature from $750 \mathrm{~K}$ to $300 \mathrm{~K}$. For a cluster of size $N$ we lower the temperature by $1 \mathrm{~K}$ for every $5+N / 2$ time steps of length 24 fs. The time step is too large to have accurate energy conservation during the optimization. This can cause unrealistic behavior when the atoms move quickly, but is not likely to affect the results of an annealing where the result is mostly determined at lower temperatures. The optimization is performed with a very coarse grid spacing of $0.24 \AA$.

At the end of the MD simulation we perform a structure optimization with normal DFT parameters using the BFGS algorithm such that the structure is guaranteed to be a local minimum.

These optimizations produce planar and tetrahedral structures in qualitative agreement with previous findings, ${ }^{6,28-30}$ while larger structures tend to be irregular but with some well-formed facets. Due to the short annealing times, the larger structures are unlikely to be global optima. Figure 1 shows the 20-atom tetrahedron and the 58-atom cluster obtained with this method. Notice on the 58-atom cluster the imperfect fivefold symmetry center reminiscent of those found on icosahedral clusters.

\section{B. Simulated annealing with EMT}

For a larger range of clusters $(N=6-200)$ we calculate structures using a simple EMT potential ${ }^{15,16}$ implemented in ASAP. ${ }^{31}$ This potential is designed to provide reasonable descriptions of elastic and cohesive properties. It is a classical potential and as such contains no explicit description of electronic behavior.

For each size of cluster we perform a simulated annealing wherein the temperature varies from above the melting point (1337 K for $\mathrm{Au}$ ) to $200 \mathrm{~K}$ with $200 \mathrm{MD}$ steps of $6.0 \mathrm{fs}$ for each $1 \mathrm{~K}$ decrease in temperature. The resulting structures frequently have fivefold symmetry centers surrounded by 111 facets, resembling partially formed icosahedra or decahedra.
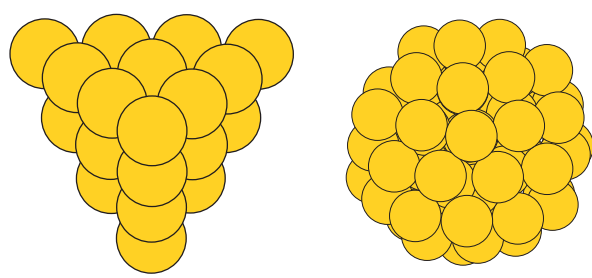

FIG. 1. (Color online) 20- (left) and 58-atom (right) clusters obtained by simulated annealing with DFT. 

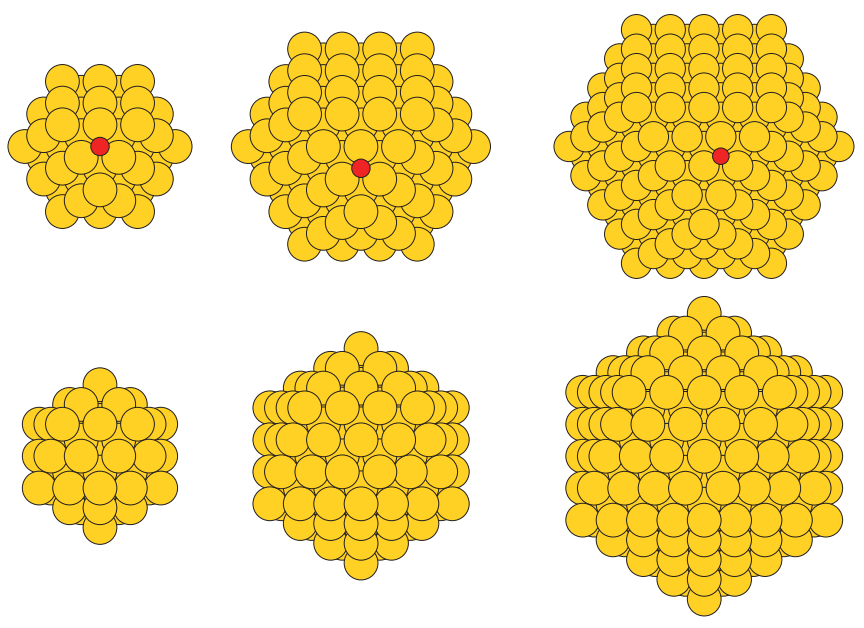

FIG. 2. (Color online) Cuboctahedra (top) and icosahedra (bottom) with 55, 147, and 309 atoms. For the cuboctahedra, an $\mathrm{O}$ atom is shown at the fcc site closest to the center of an 111 facet.

Again, at the end of the simulated annealing we perform a BFGS structure optimization with the usual DFT parameters.

\section{Atomic shell structures}

Finally, we consider the cuboctahedral and icosahedral series of structures. Each cluster can be constructed geometrically from the previous one by adding one complete shell of atoms. The cuboctahedra and icosahedra have closed atomic shells at the same numbers (see Fig. 2). The first few geometric shell closings are $N=13,55,147,309$, and 561 .

We would like to study the chemical properties of clusters by calculating adsorption energies of atoms on clusters of different sizes. A systematic comparison can be made if we ensure that the local geometry around the adsorbate remains identical for all clusters independent of size.

For the cuboctahedra we generate clusters with different numbers of atoms by stripping off atoms one by one from one cuboctahedron until only the next-smaller cuboctahedron remains. For each step, the next atom to be removed is chosen at random among those that have the lowest coordination numbers and are not part of the smaller cuboctahedron. This procedure is shown in Fig. 3.

As mentioned, to obtain adsorption energies that can be meaningfully compared across the different clusters, we must avoid changing the immediate environment of the adsorbate when removing atoms. For this reason we do not simply remove the outermost shell. Instead, we choose an adsorption site on a particular facet, then remove atoms as necessary on the other sides of the cluster such that the local facet is changed only minimally.

This method preferentially strips off corner atoms and atoms on the most open facet, opening a new facet only when necessary. This avoids very unphysical geometries. We run the calculations multiple times using a pseudorandom number generator with different random seeds, yielding a small ensemble that shows the dependence of cluster properties on the randomization.

A similar procedure can be applied to the icosahedra. However, in the icosahedra, the distance between atoms
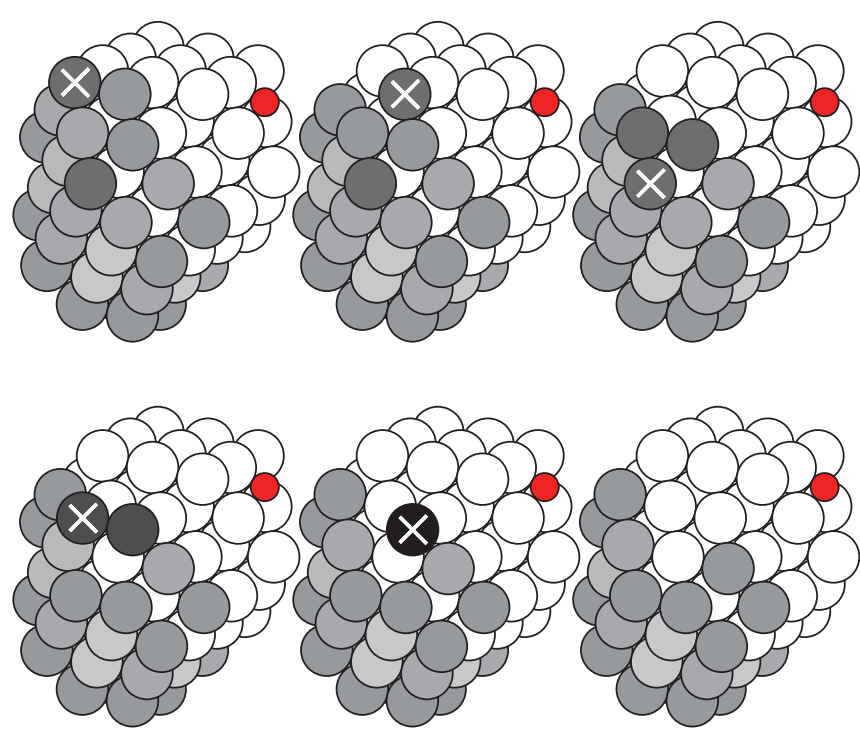

FIG. 3. (Color online) Generation of regular clusters with different numbers of atoms. The white atoms belong to the 55-atom cuboctahedron, while the gray atoms are stripped off one by one as marked with a cross. Removable atoms with lower coordination numbers have darker shades of gray, and at each step one of the lowest-coordinated atoms is removed at random. An oxygen atom (small red circle) is shown at the adsorption site.

in successive layers is different from the distance between atoms within the same layer, which means the local geometry around an adsorbate cannot always be preserved as for the cuboctahedra.

\section{INTRINSIC PROPERTIES OF CLUSTERS}

In the following we study energies and electronic properties of the different types of clusters. Note that all clusters have been relaxed using DFT such that all structures are local minima corresponding to the same force method, and have directly comparable total energies.

\section{A. Cluster structure and stability}

Figure 4 compares the energy per atom for Au clusters of the different types. EMT-optimized and regular clusters have been generated multiple times from different pseudorandom number sequences, yielding four data points for each cluster size. The DFT-optimized structures generally have energies lower than or equal to the other methods followed by EMT. Among the regular structures, icosahedra usually have lower energies than cuboctahedra. Even where the regular clusters have closed geometric shells $(N=55$ and 147), they are less stable than the structures obtained by simulated annealing with EMT.

Prominent kinks in the energy are visible around $N=34$, 58, and 92 atoms. These are "magic numbers" corresponding to major electronic shell closings. They are well known in the jellium cluster models of simple metals ${ }^{32-34}$ and have also been observed in mass spectra of noble-metal clusters. ${ }^{35}$ The kinks in energy due to electronic shell structure are robust enough to be visible for all types of clusters considered. Figure 5 provides a closer view of the energies of smaller clusters. To improve 


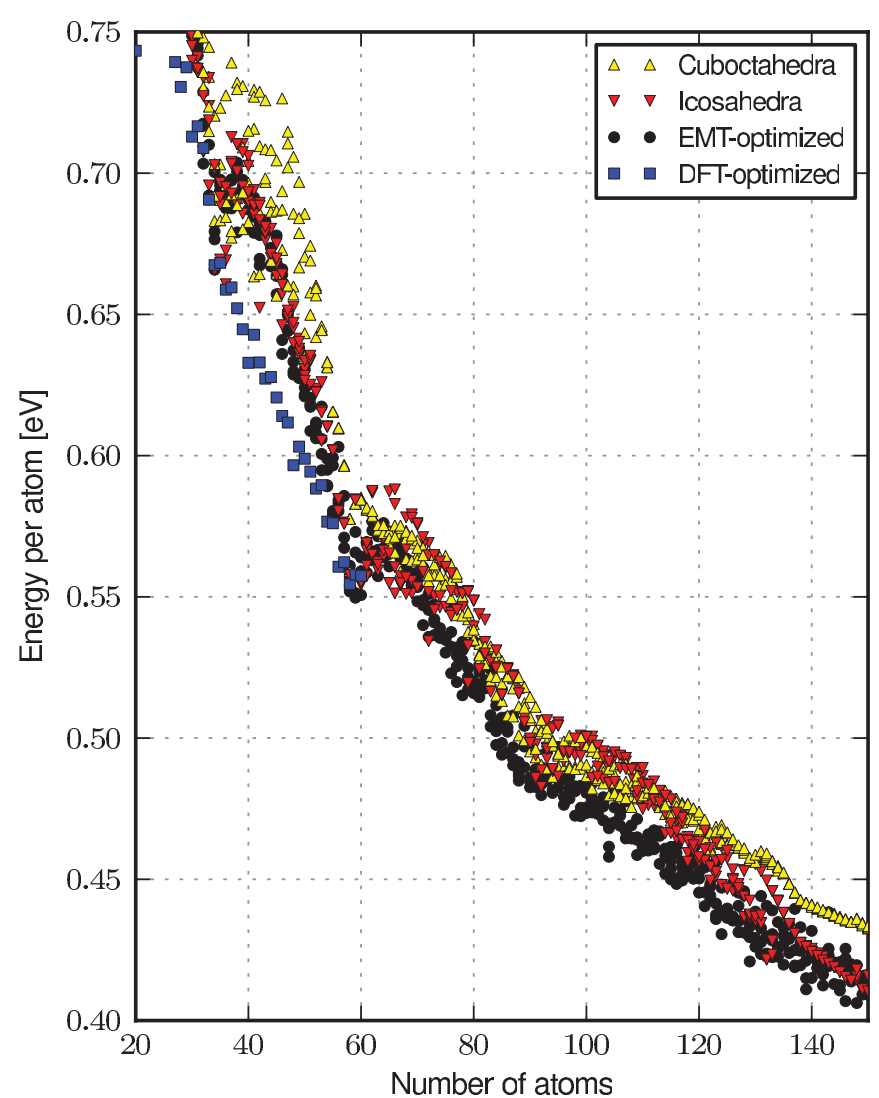

FIG. 4. (Color online) Energy per atom for different Au cluster types as a function of cluster size. The energy reference corresponds to bulk $\mathrm{Au}$.

legibility, a smooth function of $N$ of the form

$$
E_{\text {fit }}(N)=a_{0}+a_{1} N^{1 / 3}+a_{2} N^{2 / 3}+a_{3} N,
$$

is subtracted from all energies. The coefficients $\left(a_{0}, a_{1}, a_{2}, a_{3}\right)$ are obtained by fitting the energies of the DFT-optimized

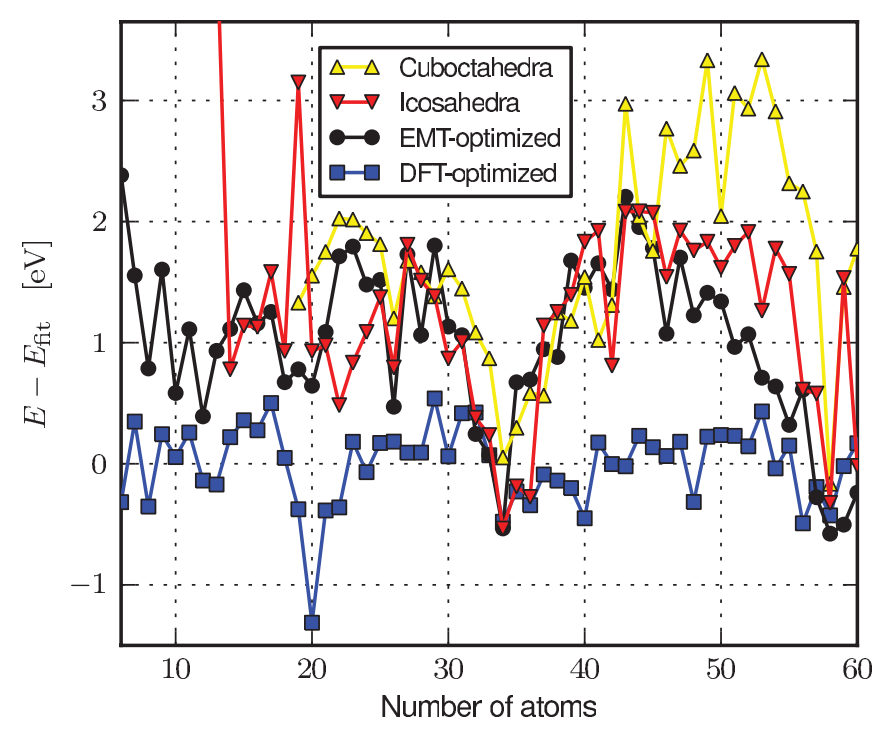

FIG. 5. (Color online) Energy for different Au cluster types as a function of cluster size $N$. A smooth function of $N$ [cf. Eq. (1)] has been subtracted from all data points for legibility.

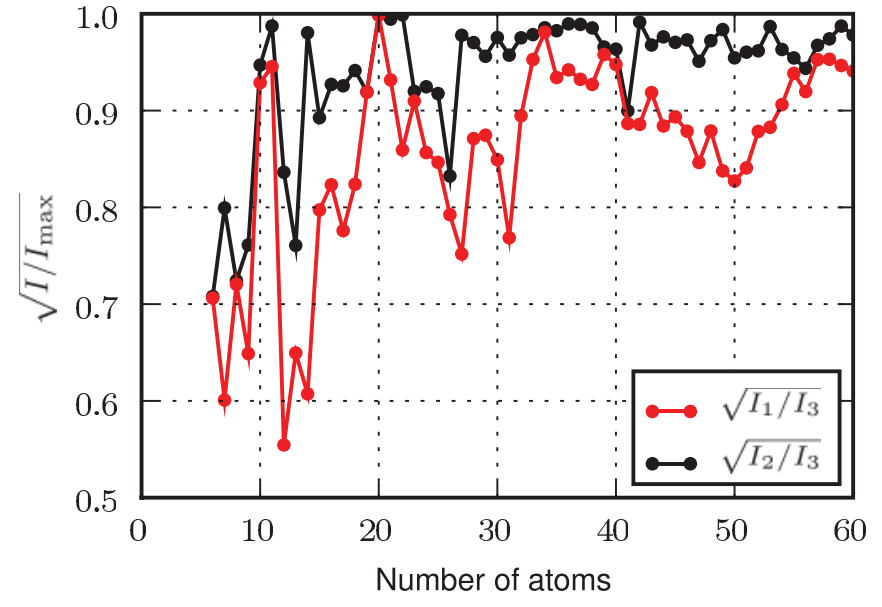

FIG. 6. (Color online) Square roots of ratios $I_{1} / I_{3}$ and $I_{2} / I_{3}$ of the three principal moments of inertia $I_{1} \leqslant I_{2} \leqslant I_{3}$ as a function of cluster size, showing deformations of the structures.

clusters. For the other kinds of clusters, only the lowest-energy data point found among four attempts is shown for each $N$.

The DFT-optimized clusters up to 13 atoms are planar except for $N=10$ and 11 . The predicted transition between planar and three-dimensional structures depends strongly on the approximation of exchange and correlation and has been studied more systematically by several authors. ${ }^{28,29}$

The particularly visible feature at $N=20$ is the well-known tetrahedron. ${ }^{36}$ Aside from this, particularly stable clusters are $N=34,40,48$, and 56/58. The EMT-based and regular structures tend to obtain comparable energies only around the major magic numbers $N=34$ and 58. In between the magic numbers, the EMT-based structures have higher energies than the DFT-optimized ones by typically $1-2 \mathrm{eV}$.

The main structural difference between the DFT-optimized clusters and other types is that the DFT-optimized clusters systematically deviate from spherical shapes when doing so is favorable to the electronic structure. A rough measure of how spherical a cluster is can be obtained by considering the moments of inertia. For each cluster the three principal moments of inertia $I_{1} \leqslant I_{2} \leqslant I_{3}$ are calculated. Figure 6 shows the ratio $\sqrt{I_{1} / I_{3}}$ and $\sqrt{I_{2} / I_{3}}$, that is, the square root of the inverse ratio between the largest principal moment and each of the smaller ones, as a function of cluster size. The most symmetric clusters are found around the magic numbers $N=20,34,40$, and 58, while intermediate clusters are deformed considerably. A similar variation has been predicted for $\mathrm{Cu}$ clusters $^{37,38}$ and in several deformable-background jellium models. ${ }^{39,40}$ Clusters just above magic numbers are from jellium models expected to be prolate while clusters below magic numbers are expected to be oblate. Such a trend is not clearly visible from our results. This is most likely due to the roughness of the optimization procedure combined with the presence of a physical atomic lattice, modifying the simple model picture.

Figure 7 compares the stabilities of $\mathrm{Cu}, \mathrm{Ag}$, and $\mathrm{Au}$ (top). For comparison, two other transition metals, $\mathrm{Pd}$ and $\mathrm{Pt}$, are also shown (bottom). For each species, the energy is calculated using simulated annealing with EMT followed by a local geometry optimization with DFT for $N=6-200$. A smooth 


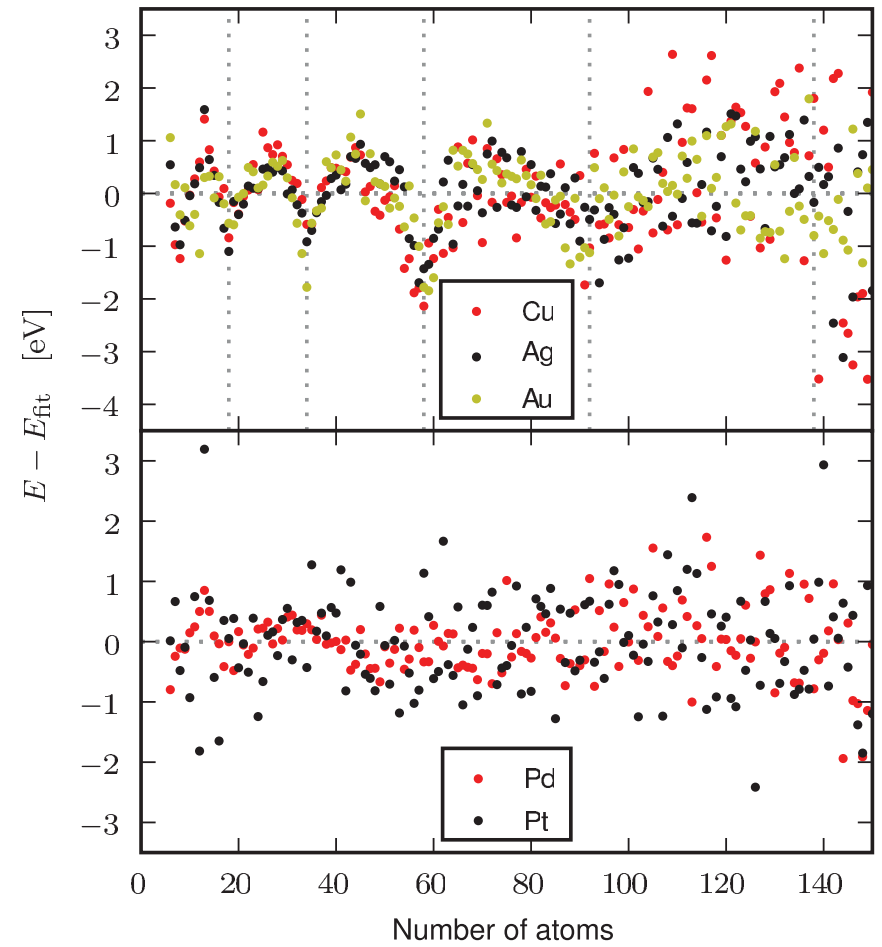

FIG. 7. (Color online) Energy minus fitted trend line for noblemetal clusters (top) and $d$-band metals (bottom). Electronic magic numbers at $N=18,34,58,92$, and 138 are indicated.

function is then subtracted by fitting the energies for each element according to Eq. (1), such that the figure shows the deviation from a smooth trend line.

As for $\mathrm{Au}$, the other noble-metal clusters are particularly stable close to the magic numbers $18,34,58$, and 92 , and to a lesser extent at 138. Deviations from the trend line oscillate with a peak-to-peak variation of around $3 \mathrm{eV}$. Beyond 138, the periodic trend is gradually obscured by fluctuations which are probably caused by imperfections in the optimization procedure.

The three noble metals exhibit roughly identical behavior except in the region $N<18$, where Au differs noticeably from $\mathrm{Cu}$ and $\mathrm{Ag}$. In this case, the Au clusters deform considerably from the geometries found by EMT, tending toward flat structures. $\mathrm{Cu}$ and $\mathrm{Ag}$ clusters remain round. The tendency of small Au clusters to form planar structures has been well documented and has been attributed to relativistic effects causing a contraction of the $s$ orbitals compared to the $d$ orbitals. ${ }^{41-43}$ The differences in behavior between noble metals here appear to be caused exclusively by the relativistic behavior of $\mathrm{Au}$, and not, for example, the location of the $d$ band in relation to the Fermi level, which would set $\mathrm{Cu}$ and $\mathrm{Ag}$ apart.

For Pd and Pt, no magic numbers are observed. Deviations from the trend line instead appear to depend on how wellformed the clusters are, for example, the type and regularity of their facets as seen from visual inspection of the cluster structures. Thus, the stability of non-noble transition-metal clusters is determined mostly by local atomic arrangement, corresponding to interactions between the short-range $d$ electrons.

\section{B. Electronic structure}

Figure 8 compares the density of states (DOS) per atom of Au (top) and Pt (bottom) clusters optimized with EMT as a two-variable function of cluster size and energy. For each cluster, the DOS is approximated as a sum of Gaussians of width $0.07 \mathrm{eV}$ centered on each energy eigenvalue.

For both $\mathrm{Au}$ and $\mathrm{Pt}$ the $d$ states very quickly form the usual continuous, narrow band which beyond $N=20$ changes only very little. The $s$ states split up into multiple electronic shells which are separated by gaps as in the jellium shell model. As $N$ increases the shells gradually broaden to form a continuous band. Oscillations in the DOS originating from the shell structure are still clearly visible even for the largest clusters.

For $\mathrm{Au}$, where the Fermi level is located well above the $d$ band, the electronic shells due to the $s$ electrons are filled one by one as cluster size increases. When one shell is full, the Fermi level jumps to the next-higher shell, causing the abrupt shifts in Fermi level and large band gaps.

The Fermi level for Pt is lodged at the top of the $d$ band where the DOS is extremely high. Therefore, no gaps or jumps

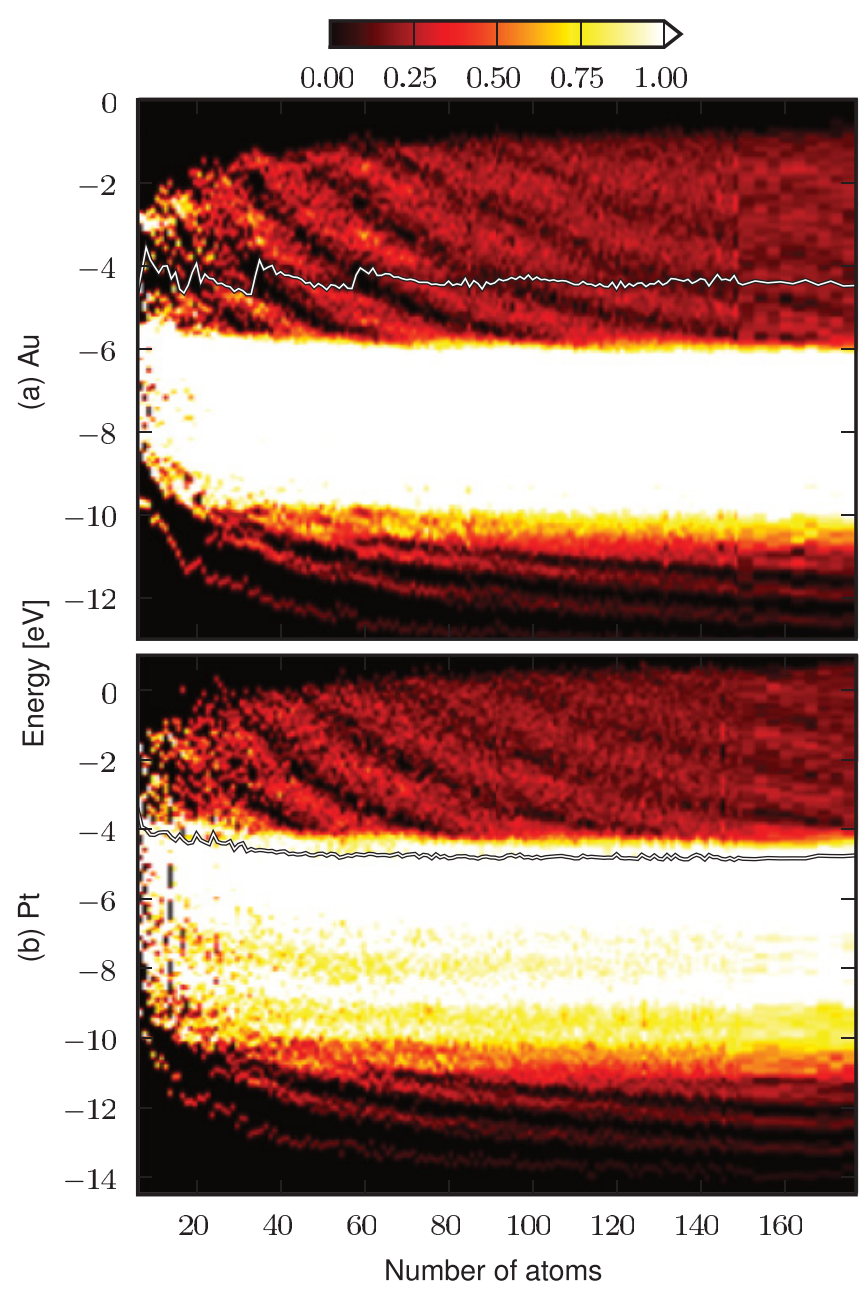

FIG. 8. (Color online) DOS in arbitrary units of EMT-optimized $\mathrm{Au}$ clusters (top) and Pt clusters (bottom) as a function of cluster size and energy. The line indicates the Fermi level. Values larger than 1.0 are truncated to 1.0 . 


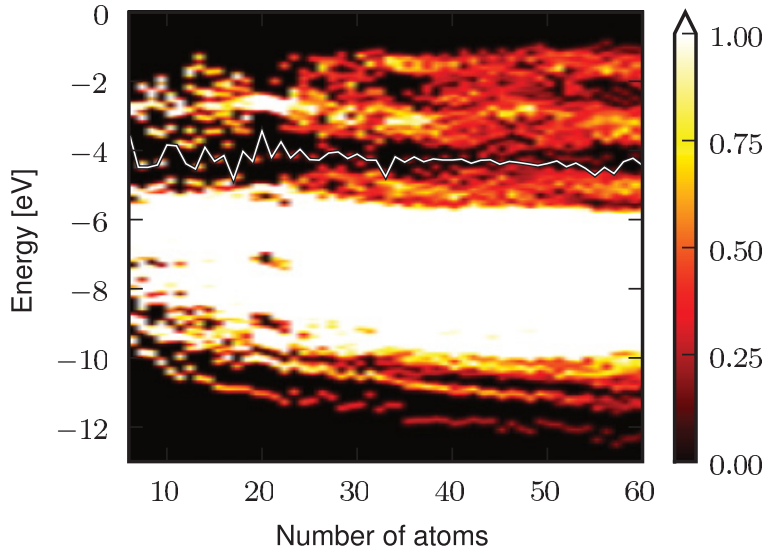

FIG. 9. (Color online) DOS in arbitrary units of DFT-optimized clusters. The line indicates the Fermi level. DOS values larger than 1.0 are truncated to 1.0 .

in the Fermi level are possible, and the cluster will not exhibit any electronic magic numbers even though the $s$ electrons form shells exactly like Au.

Comparing to the DOS of the DFT-optimized clusters on Fig. 9, the DFT-based optimization consistently creates very large gaps around the Fermi level for all small clusters. For clusters with an odd number of electrons (where the gap is zero because of spin degeneracy), a single singly occupied state is located at the middle of a symmetric gap. Similar behavior has been reported for $\mathrm{Cu}$ clusters. ${ }^{37} \mathrm{~A}$ significant difference compared to the roughly spherical clusters obtained with EMT is that the shell structure cannot easily be seen as distinct shells that move down in energy as the cluster size increases. Rather there is an accumulation of states some way above as well as below the Fermi level. Only close to the shell closings does the DOS resemble that of the EMT-optimized clusters. A consequence of this is that the abrupt jumps in Fermi level $\epsilon_{F}$ seen for EMT-optimized clusters are less visible for the DFToptimized ones. However, a significant change in ionization potential $I$ and electron affinity $A$ still accompanies a magic number. Figure 10 (a) shows the difference $-(I+A) / 2 \approx \epsilon_{F}$. The value increases sharply at each magic number.

It is easy to understand that gaps at the Fermi level are associated with an increase in stability. The gap is created so that occupied states are pushed down in energy while pushing unoccupied ones up, resulting in a decrease of band structure energy.

For larger clusters that are not close to magic numbers (e.g., $N \approx 45$ ) the gap becomes small, but a significant depletion of states around the Fermi level persists (a similar depletion of states close to the Fermi level is also seen for the EMT-optimized clusters, for example, for $N \approx 45$ and 70 ; this is a product of the local structure optimization with DFT after the EMT annealing). The combined structural and electronic trends of the DFT-optimized clusters thus point to a picture where clusters far from magic numbers will deform significantly, maximizing the gap at the Fermi level. In a sense, this deformation creates a new magic number for every size of cluster provided the clusters are small enough. As long as such a gap remains, strong even-odd oscillations of the electronic properties will persist due to the singly filled state
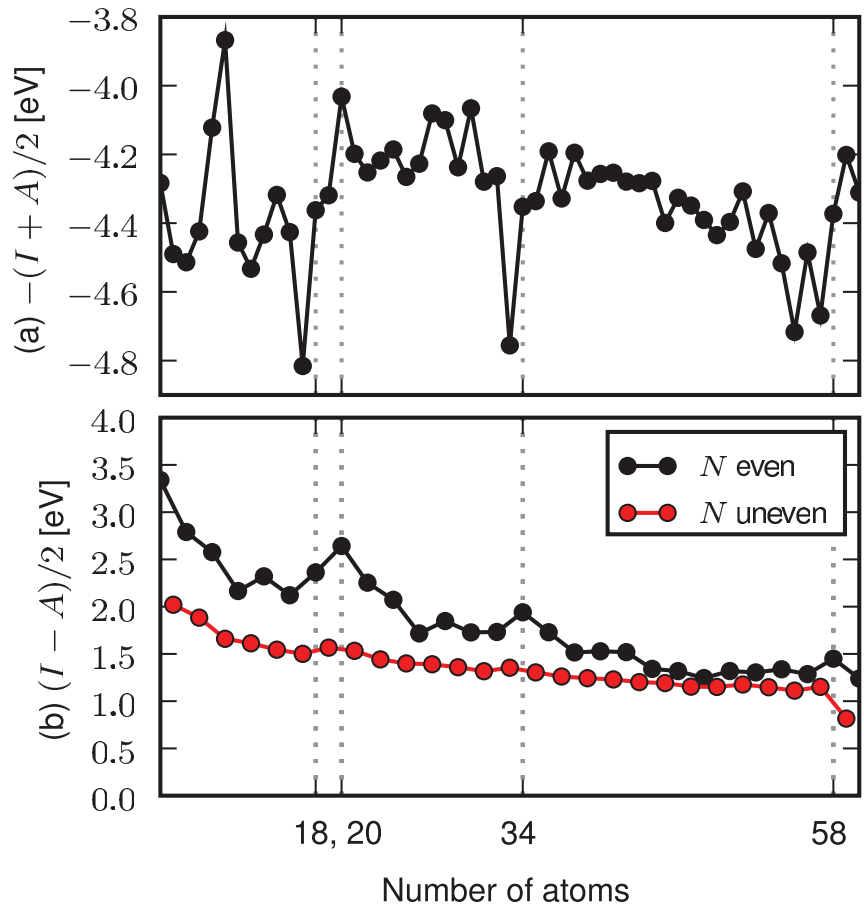

FIG. 10. (Color online) Fermi level (top) and chemical hardness (bottom) calculated from ionization potential $I$ and electron affinity $A$ for DFT-optimized Au clusters as a function of cluster size.

in uneven clusters. Figure 10(b) shows the band gap calculated as $(I-A) / 2$ as a function of cluster size. Even and odd clusters are plotted as separate lines. The structure optimization tends to obtain larger gaps close to the spherical shell closings, and so the even-odd alternations are larger close to these. The even-odd alternations become small compared to the $0.1-\mathrm{eV}$ smearing for clusters larger than $\approx 40$ atoms except at the electronic shell closings.

Figure 11 compares the calculated DOS near the Fermi level for EMT-optimized, icosahedral and cuboctahedral structures. The structures yield remarkably similar electronic shells separated by gaps.

Similarities between the electronic shell structures of spherical and faceted structures with hundreds of electrons have previously been found in the context of well potentials. ${ }^{44}$ Our results show that the inclusion of a $d$ band, as well as the inclusion of an atomic lattice with various irregularies as per the different cluster generation procedures used here, have limited effect on the shell structure in this size range.

The highly visible change for icosahedra close to $N=130$ happens when a sufficiently large number of atoms have been removed from the same side of the cluster, causing a substantial collective movement of the surrounding atoms (this is, therefore, just an artifact of the cluster generation method).

\section{CHEMICAL PROPERTIES OF CLUSTERS}

In this section we consider the chemisorption of various atoms on cuboctahedral clusters.

Adsorption energies are calculated as follows. A series of cuboctahedral clusters is generated by the procedure described in Sec. IIIC, so as to preserve the local geometry around 


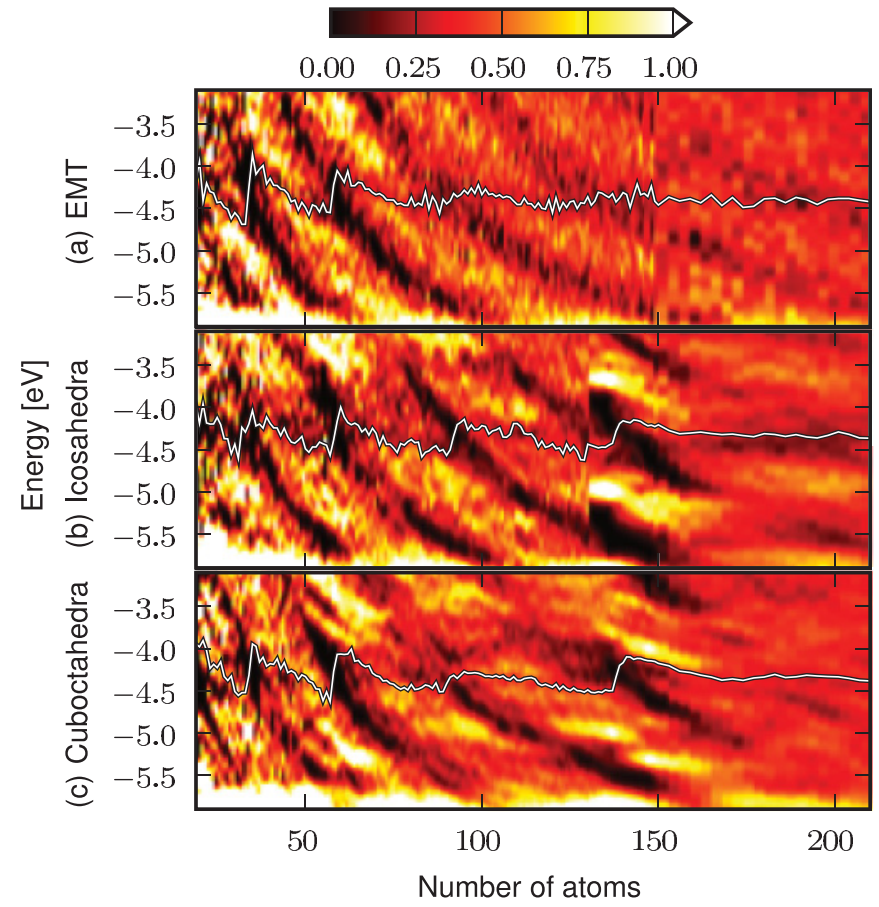

FIG. 11. (Color online) DOS near Fermilevel for different types of clusters. (a) Clusters optimized using the EMT potential. (b) Clusters based on icosahedra. (c) Clusters based on cuboctahedra. Units are arbitrary.

the desired adsorption site. A structure optimization is then performed on the entire cluster plus adsorbate, yielding a total energy of the combined system. From this energy we subtract the energies of the isolated adsorbate and the isolated cluster. Ideally, the energy of the isolated cluster should be calculated by removing the adsorbate, then rerelaxing the structure. However, this is likely to cause atoms to move significantly for at least some of the smaller clusters, obscuring the general trends that we are trying to examine without providing any insight. For this reason we instead neglect to rerelax the clusters after removing the adsorbate. Calculated binding energies therefore tend to be overestimated.

Figure 12(a) shows the adsorption energy of oxygen on cuboctahedral clusters as a function of cluster size for two different adsorption sites. One is the fcc site as close as possible to the center of an (111) facet, which locally resembles a (111) surface. The other is the hcp site closest to the corner of a (111) facet, where $\mathrm{O}$ frequently binds more strongly. For each site there are four series of data points corresponding to different random seeds in the cluster generation procedure.

Adsorption energies on both sites are related to the distribution of the electronic shell closings $N=34,58,92$, and 138, where binding energies are particularly low. Near the geometric shell closings 55 and 147, where the clusters are regular and closer to being spherical, this behavior is most pronounced. The change near 92 is less abrupt, and we attribute this to the less symmetric structures generated far from geometric shell closings (we believe that 92 would stand out more clearly for clusters with more realistic geometry).

Consider the behavior at the magic number 138. The binding gradually weakens until 138, after which it abruptly
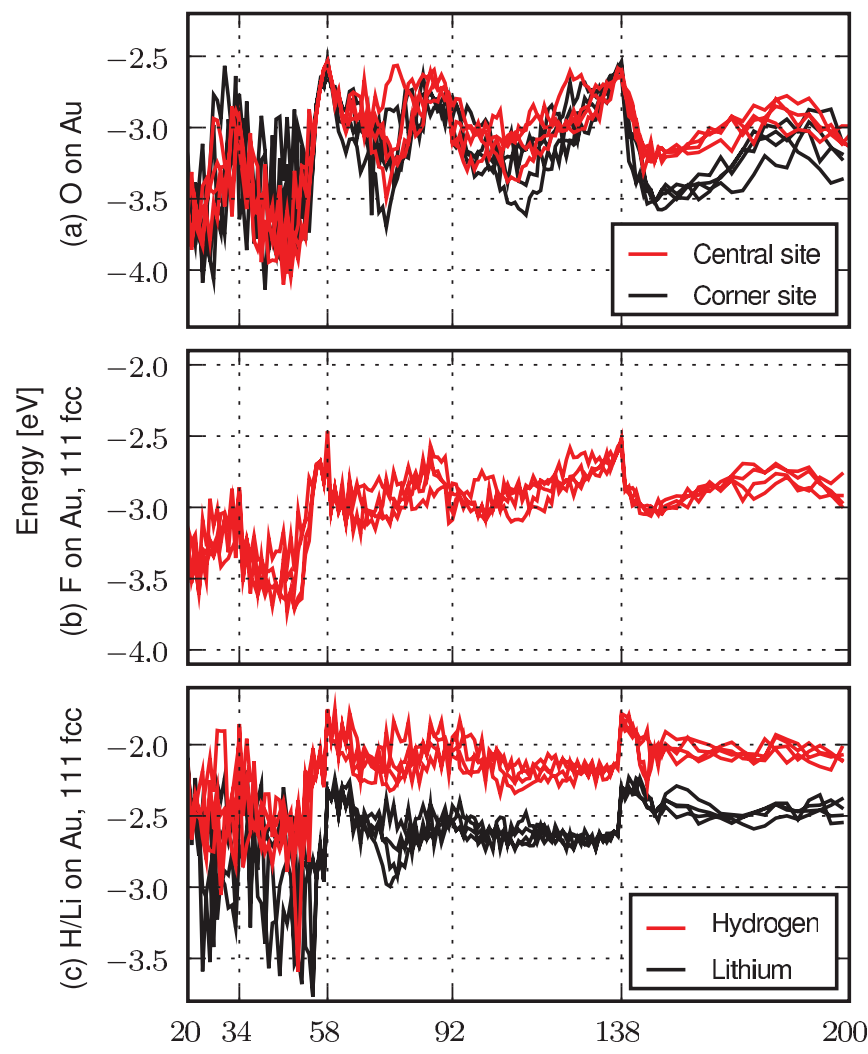

FIG. 12. (Color online) Adsorption energies as a function of $\mathrm{Au}$ cluster size. (a) $\mathrm{O}$ on the fcc site closest to the center of an (111) facet and the hcp site at the corner of an (111) facet. (b) F on the central (111) fcc site. (c) $\mathrm{H}$ and $\mathrm{Li}$.

changes from very weak to very strong. The same effect is seen to a smaller extent at 58 (the preceding weakening of binding energy is in this case not gradual, but coincides with the completion of a local facet, as discussed below).

Clusters slightly larger than a magic number will have one or more loosely bound electrons which can easily be donated to O. Clusters can in this sense be characterized as alkalimetal-like, noble-metal-like, or halogenlike depending on their location relative to magic numbers. What the comparison between the two adsorption sites shows is that the main variation of adsorption energy due to the electronic shell structure is not strongly affected by local geometry around the adsorbate. While there are intriguing differences between the binding on the central site and the corner site, such details are probably too specific to make predictions about more realistic geometries.

The alkali-metal-like or halogenlike behavior of clusters near magic numbers is demonstrated in Figs. 12(b) and 12(c), which show the adsorption energy of $\mathrm{F}, \mathrm{H}$, and Li on the central (111) fcc site as a function of cluster size.

The variation of F adsorption energy [Fig. 12(b)] around magic numbers is qualitatively similar to that of $O$. Since $F$ is more electronegative, the increase in binding energy past a magic number is more abrupt and is clearly visible for all the magic numbers 34, 58, 92, and 138. However, F can accept only one electron, and so the total magnitude of the increase in energy near $N=58$ and $138(0.5 \mathrm{eV})$ is smaller than in the case of $\mathrm{O}$ (up to $1.0 \mathrm{eV}$ ). The variation of $\mathrm{F}$ binding energy 
at magic numbers is roughly equal to the increase in Fermi energy of the cluster $(0.6 \mathrm{eV})$.

The electropositive Li shows the opposite trend: A steep decrease in binding energy follows a magic number. Again, the change in binding energy is close to $0.5 \mathrm{eV}$ corresponding to the sharing of one electron. Hydrogen somewhat surprisingly follows the same trend as $\mathrm{Li}$, even though the $\mathrm{H} 1 s$ state is approximately as low lying as the $\mathrm{O} 2 p$ states. We analyze this further in the next section.

Apart from the variation due to magic numbers, binding energies of all species tend to be stronger for the smallest clusters $(N<50)$. The very steep change in binding energy around $N=50$ which is seen for all adsorbates can be attributed to geometric changes of the local facet. The triangular 6-atom (111) facet of the 55-atom cuboctahedron appears to be generally unreactive, as all four adsorbates bind weakly to it, including $\mathrm{O}$ on both the central site and the corner site. Several previous works have noted that a lower overall coordination of nearby $\mathrm{Au}$ atoms has been found to increase binding strengths. ${ }^{27,45,46}$ The effects due to local geometry are, however, comparatively small for Au clusters larger than 55 atoms. The global electronic shell structure is responsible for most of the variation in adsorption energy, as evidenced by the consistent oscillating trend.

For comparison, adsorption energies of $\mathrm{O}$ on $\mathrm{Pt}$ clusters are shown on Fig. 13. The clusters have the same initial structures as the Au clusters in Fig. 12(a). Instead of a smooth oscillating trend, the binding energy can vary significantly when nearby facets are modified, even though the modification takes place several sites away. This causes the adsorption energy to depend much more sensitively on the geometry of the nearby facets. Apart from the strongly geometry-dependent variations, an overall decrease in $\mathrm{O}$ binding energy with size is also observed, which resembles that of Au. We note that the changes in adsorption energy on Au clusters occur even when atoms are added on the directly opposite side of the cluster as seen from the adsorption site.

Let us return to the Au clusters. The projected density of states (PDOS) on the adsorbate reveals useful information on how the atomic states hybridize with the clusters. The PDOS as a function of energy $\epsilon$ on atom $A$ is calculated from the atomic expansion of the Kohn-Sham eigenstates $\left|\tilde{\psi}_{n}\right\rangle$ and eigenvalues $\epsilon_{n}$ as

$$
\rho_{A}(\epsilon)=\sum_{n}\left\langle\tilde{\psi}_{n}\left|P_{A}\right| \tilde{\psi}_{n}\right\rangle \delta\left(\epsilon-\epsilon_{n}\right),
$$

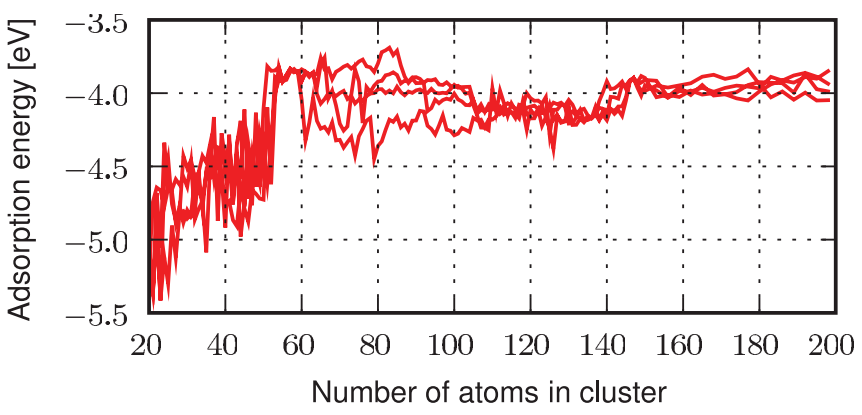

FIG. 13. (Color online) Adsorption energy of $\mathrm{O}$ on $\mathrm{Pt}$ as a function of cluster size.

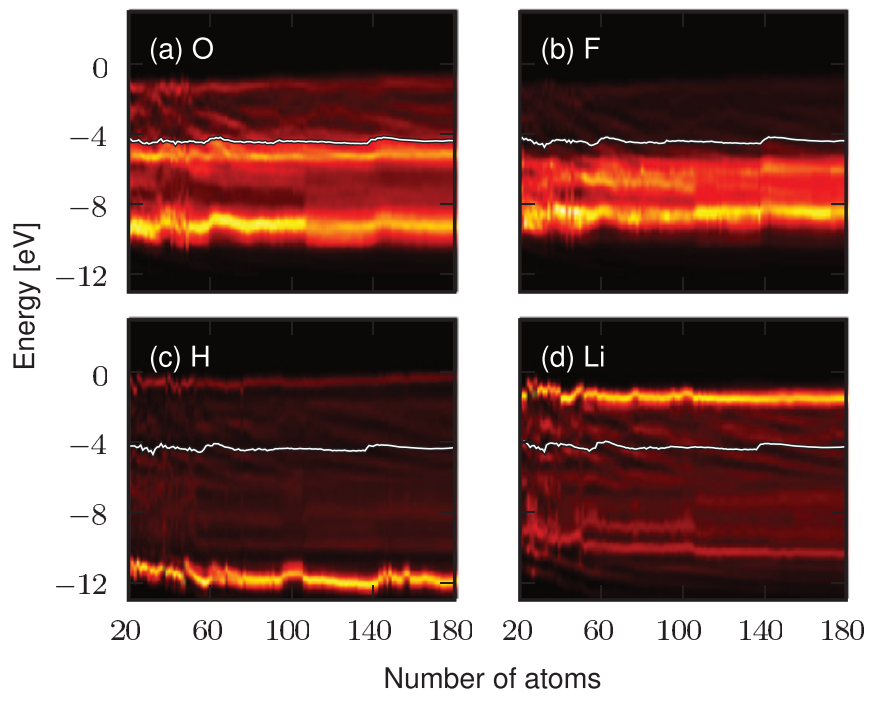

FIG. 14. (Color online) Projected density of states on O, F, H, and $\mathrm{Li}$ adsorbed on Au clusters as a function of cluster size and energy calculated using the atomic basis set. The white line indicates the Fermi level. Units are arbitrary.

where

$$
P_{A}=\sum_{a \in A, b \in A}\left|\Phi_{a}\right\rangle\left(S_{A}^{-1}\right)_{a b}\left\langle\Phi_{b}\right|
$$

is a projection operator onto the subspace spanned by the basis functions $\left|\Phi_{a}\right\rangle$ on atom $A$, and $\left(S_{A}\right)_{a b}=\left\langle\Phi_{a} \mid \Phi_{b}\right\rangle$ is the overlap matrix within that subspace.

Figure 14 shows the PDOS on the O, F, H, and Li atoms adsorbed on $\mathrm{Au}$ cuboctahedra of varying size. For $\mathrm{O}$ the $p$ states split into bonding and antibonding states at the top and bottom of the $d$ band (see also Ref. 47 on hybridization on $\mathrm{Au}$ surfaces). Both bonding and antibonding states are occupied, and they remain qualitatively similar for all sizes of clusters although some variation is seen around the magic numbers. The weights of bonding and antibonding states can change drastically when the local geometry is modified, but for clusters larger than 55 atoms these changes are not reflected strongly in the adsorption energy and do not explain the trends (for example, the most visible change, around $N=105$, occurs when the last two second-nearest-neighbor atoms are added. This causes the adsorption energy to change by $0.15 \mathrm{eV}$ ). The behavior of $\mathrm{F}$ resembles that of $\mathrm{O}$ except the coupling is weaker. For $\mathrm{H}$ a very low-lying state is created at the bottom of the $s$ band. The surprising formation of such a low-lying state at the bottom of the band has been described previously for $\mathrm{H}$ adsorption on $\mathrm{Mg}$ surfaces. ${ }^{48}$ Finally, Li has a high-lying state which is above the Fermi level.

\section{NEWNS-ANDERSON MODEL}

The peculiar properties of metal nanoparticles are sometimes attributed to the discrete spectrum causing the icle to behave like a molecule rather than a bulk material characterized by a continuous spectrum. However, the DOS quickly $(N>$ a few dozen atoms) becomes effectively continuous on any reasonable energy scale $(\sim 0.1 \mathrm{eV})$. The primary feature distinguishing clusters with a few dozen 
to a few hundred atoms compared to bulk is not whether the DOS is discrete or continuous, but rather the fact that the approximately continuous DOS remains grouped into shells separated by gaps. The size-dependent variation in this effectively continuous DOS and, in particular, the distribution of magic numbers are the significant factors that make clusters with many hundreds of atoms still differ from bulk Au. Since the DOS of an Au cluster larger than a few dozen atoms can effectively be regarded as continuous, we in the following apply the Newns-Anderson model ${ }^{49}$ to understand chemisorption on Au clusters. The Newns-Anderson model considers a single state $|a\rangle$ on an atom which hybridizes with a continuum of states $\{|k\rangle\}$ of the metal surface described by a Hamiltonian of the form

$$
H=H_{0}+V,
$$

where $H_{0}$ is the Hamiltonian of the uncoupled cluster plus adsorbate and $V$ describes the coupling. In the basis consisting of metal eigenstates $\{|k\rangle\}$ plus adsorbate eigenstate $|a\rangle$, the uncoupled Hamiltonian $H_{0}$ is diagonal, and the NewnsAnderson Hamiltonian can be written in block form as

$$
H=\left[\begin{array}{ccc|c}
\ddots & & 0 & \vdots \\
& \epsilon_{k} & & v_{a k}^{*} \\
0 & & \ddots & \vdots \\
\hline \cdots & v_{a k} & \cdots & \epsilon_{a}
\end{array}\right] .
$$

Here $\epsilon_{a}$ and $\epsilon_{k}$ are the uncoupled energy eigenvalues on the atom and in the metal while $v_{a k}=\langle a|V| k\rangle$ are coupling matrix elements.

Our idea now is to perform a DFT calculation to obtain a Hamiltonian matrix of the composite system consisting of both cluster and adsorbate, then transform it to Newns-Anderson form [cf. Eq. (5)]. In the basis of atomic orbitals used by GPAW, the Kohn-Sham equations are solved as a generalized eigenvalue problem, ${ }^{21}$

$$
\sum_{\nu} H_{\mu \nu} c_{\nu n}=\sum_{\nu} S_{\mu \nu} c_{\nu n} \epsilon_{n} .
$$

The overlap matrix $S_{\mu \nu}$ is present because the basis is nonorthogonal. The Hamiltonian can be regarded as a blocked matrix

$$
H^{\mathrm{DFT}}=\left[\begin{array}{cc}
H^{M} & H^{M A} \\
H^{A M} & H^{A}
\end{array}\right],
$$

with one block $H^{M}$ corresponding to the metal or cluster, one block $H^{A}$ corresponding to the atom, and the offdiagonal blocks $H^{M A}, H^{A M}$ corresponding to the interaction. We diagonalize each of the metallic and atomic submatrices according to

$$
\begin{aligned}
\sum_{\nu} H_{k^{\prime} \nu}^{M} c_{\nu k}^{M} & =\sum_{\nu} S_{k^{\prime} \nu}^{M} c_{v k}^{M} \epsilon_{k}, \\
\sum_{v} H_{a^{\prime} \nu}^{A} c_{v a}^{A} & =\sum_{v} S_{a^{\prime} v}^{A} c_{v a}^{A} \epsilon_{a},
\end{aligned}
$$

to obtain values for the energies $\epsilon_{k}$ and $\epsilon_{a}$ of the uncoupled systems. Clearly, these are approximate, as the real energy values could be evaluated self-consistently on each of the uncoupled systems using a separate DFT calculation. However, the Hamiltonian and eigenvalues from one self-consistent calculation cannot be expected to be "compatible" with those from a different self-consistent calculation. Indeed, it is known from the force theorem ${ }^{15,50}$ that the first-order change in energy due to a small perturbation of the Hamiltonian is equal to the change in band structure energy, keeping the potential and density fixed. Different Hamiltonian matrices based on DFT can therefore be expected to contain all information provided that they are constructed from the same potential and density.

Using the matrices $c^{M}$ and $c^{A}$, coupling elements can be obtained through the transformation

$$
\begin{aligned}
v_{a k} & =\sum_{a^{\prime} k^{\prime}} c_{a^{\prime} a}^{A *} H_{a^{\prime} k^{\prime}}^{A M} c_{k^{\prime} k}^{M}, \\
s_{a k} & =\sum_{a^{\prime} k^{\prime}} c_{a^{\prime} a}^{A *} S_{a^{\prime} k^{\prime}}^{A M} c_{k^{\prime} k}^{M} .
\end{aligned}
$$

Thereby we have all the parameters in the Newns-Anderson Hamiltonian [Eq. (5)], although the adsorbate state has an overlap $s_{a k}=\langle a \mid k\rangle$ with each of the metallic eigenstates, meaning the basis set is nonorthogonal. Below we use expressions derived by Grimley ${ }^{51}$ for the nonorthogonal case.

If the adsorption induces a change $\delta \rho(\epsilon)$ in the metallic DOS, the adsorption energy can be written as

$$
E_{\text {ads }}=2 \int_{-\infty}^{\epsilon_{F}} \delta \rho(\epsilon) \epsilon \mathrm{d} \epsilon-\Delta N \epsilon_{F}+n_{a}\left(\epsilon_{F}-\epsilon_{a}\right),
$$

where $\epsilon_{F}$ is the Fermi level. The first term is the contribution to the binding energy from hybridization with the adsorbate (the factor of 2 assumes that each spin hybridizes equally and independently). The integral of the induced DOS,

$$
\Delta N=2 \int_{-\infty}^{\epsilon_{F}} \delta \rho(\epsilon) \mathrm{d} \epsilon,
$$

is the change in number of electrons below the Fermi level. If this integral is nonzero, either too much or too little charge will be counted in the integration up to the Fermi level, and the extra or missing electrons must then be deposited onto or taken from the Fermi level. This electron count correction is the second term, $\Delta N \epsilon_{F}$. Finally, if the atom contributes $n_{a}$ electrons which come from the adsorbate level $\epsilon_{a}$, this amount of extra charge must, in turn, be deposited on the Fermi level $\epsilon_{F}$ (last term). In the Newns-Anderson model, the first two terms of Eq. (12) are expressed as the integral over a function $\eta(\epsilon)$ such that

$$
E_{\mathrm{ads}}=\frac{2}{\pi} \int_{-\infty}^{\epsilon_{F}} \eta(\epsilon) \mathrm{d} \epsilon+n_{a}\left(\epsilon_{F}-\epsilon_{a}\right),
$$

where

$$
\begin{gathered}
\tan \eta(\epsilon)=\frac{\Delta(\epsilon, \epsilon)}{\epsilon-\epsilon_{a}-\Lambda(\epsilon)}, \\
\Lambda(\epsilon)=\frac{1}{\pi} \int_{-\infty}^{\infty} \frac{\Delta\left(\epsilon, \epsilon^{\prime}\right)}{\epsilon-\epsilon^{\prime}} \mathrm{d} \epsilon^{\prime}, \\
\Delta\left(\epsilon, \epsilon^{\prime}\right)=\pi \sum_{k}\left|\epsilon s_{a k}-v_{a k}\right|^{2} \delta\left(\epsilon^{\prime}-\epsilon_{k}\right) .
\end{gathered}
$$

The one-variable function $\Delta(\epsilon) \equiv \Delta(\epsilon, \epsilon)$ is referred to as the chemisorption function and plays the role of a continuous coupling matrix element. $\eta(\epsilon)$ is the phase shift of the complex 
self-energy $\Lambda(\epsilon)-i \Delta(\epsilon)$ and is related to the the induced DOS by

$$
-\frac{1}{\pi} \frac{\mathrm{d} \eta(\epsilon)}{\mathrm{d} \epsilon}=\delta \rho(\epsilon) .
$$

The PDOS on the adsorbate can be written as

$$
\rho_{a}(\epsilon)=\frac{1}{\pi} \frac{\Delta(\epsilon)}{\left[\epsilon-\epsilon_{a}-\Lambda(\epsilon)\right]^{2}+\Delta^{2}(\epsilon)} .
$$

Because of the approximations used in this method, calculated binding energies are by themselves not useful (or accurate) compared to the DFT results. The strength of this method lies in the conceptual simplification that the binding energy can be understood from continuous functions such as $\Delta(\epsilon)$ and $\eta(\epsilon)$. This allows the origin of the coupling and binding energy to be attributed to particular states in the cluster. Next we apply this to $\mathrm{H}, \mathrm{O}, \mathrm{Li}$, and $\mathrm{F}$ on a 58 -atom Au cluster to understand the effect of magic numbers on chemisorption.

\section{A. Adsorption of $\mathbf{H}$}

We perform a DFT calculation on a 58-atom Au cluster with $\mathrm{H}$ adsorbed to obtain the Hamiltonian and overlap matrix. In this calculation, only the $1 s$ basis function is included on $\mathrm{H}$, but otherwise the parameters are identical to those used in earlier calculations. For the $1 s$ basis function we calculate $\Delta(\epsilon)$ and $\Lambda(\epsilon)$, which is shown on Fig. 15(a) together with the line $\epsilon-\epsilon_{a}$. When $\epsilon-\epsilon_{a}=\Lambda(\epsilon)$ and $\Delta(\epsilon)$ is small, there will be resonances in $\rho_{a}(\epsilon)$ [as per Eq. (19)] corresponding to states on the atom. The adsorbate level $\epsilon_{a}$ (circle) and Fermi level (dotted line) are indicated. The resulting PDOS is shown on Fig. 15(b) together with the total DOS of the Au cluster. This

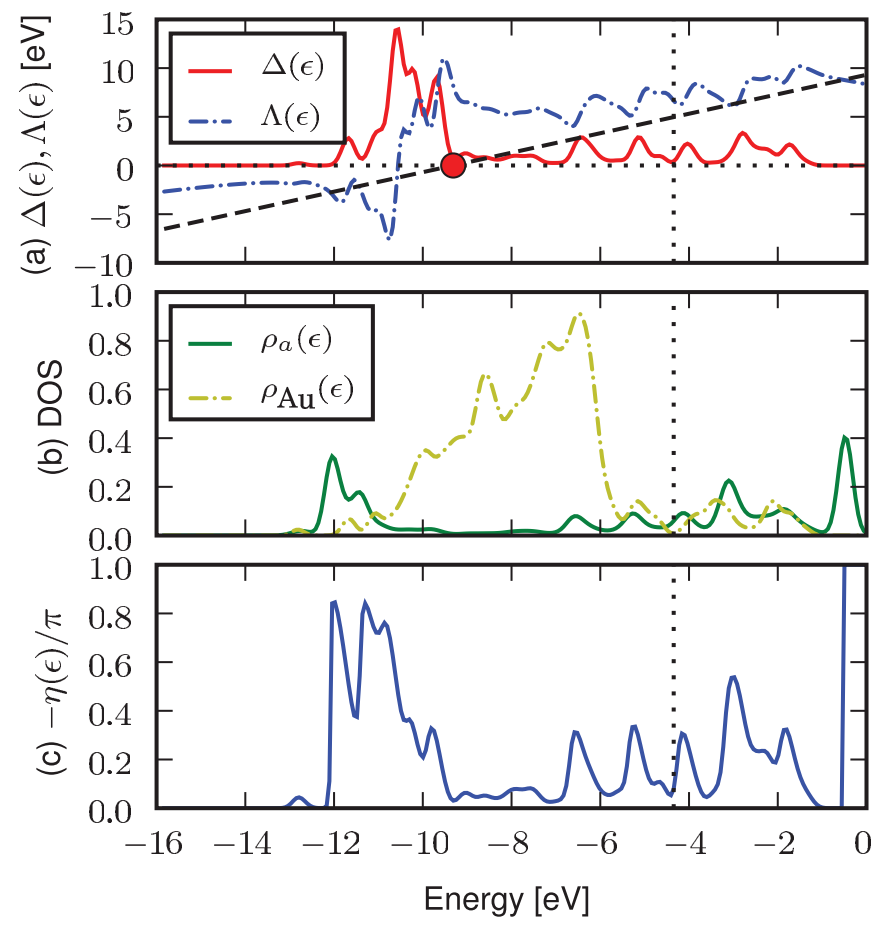

FIG. 15. (Color online) (a) $\Delta(\epsilon), \Lambda(\epsilon)$, and $\epsilon-\epsilon_{a}$ for $\mathrm{H}$ on a 58-atom Au cluster. The dotted line indicates the Fermi level. (b) Projected DOS on atom in $\mathrm{eV}^{-1}$ and total DOS of isolated cluster in arbitrary units. (c) Cumulative induced DOS. reveals that it is the strong coupling to low-lying metallic states $(\epsilon \approx-11 \mathrm{eV})$, which gives rise to a bonding, localized state at the bottom of the Au $s$ band, at $-12 \mathrm{eV}$, and an antibonding state consisting of several peaks mostly above the Fermi level.

On a side note, the very high PDOS at the antibonding state may seem surprising. Given that the adsorbate level $\epsilon_{a} \approx$ $-9 \mathrm{eV}$ is much closer to the bonding state, the bonding state would be expected to be similar to $|a\rangle$ and thus have a high PDOS on the atom, while the antibonding state should be more similar to the metallic states and therefore have a low PDOS on the atom. However, the inclusion of overlap $s_{a k}$ in the model causes part of the states on the neighboring metal atoms to be counted in the adsorbate PDOS, contributing to the prominence of the antibonding peak. While the overlap affects the calculated PDOS, the overlap is correctly taken into account in binding energies and other parts of the formalism.

In the creation of bonding and antibonding states the original adsorbate state has been eliminated, and a change $\delta \rho(\epsilon)$ in DOS has been induced in the cluster. The cumulative induced DOS $-\eta(\epsilon) / \pi$ is shown on Fig. 15(c). While the newly created bonding state at $-12 \mathrm{eV}$ can accept a certain amount of charge, a similar amount of charge has been removed from the remainder of the cluster DOS (mostly around $-10 \mathrm{eV}$ ) such that the total integral of the induced DOS up to the Fermi level is zero. The extra electron from the $\mathrm{H}$ atom is therefore deposited on the Fermi level. A higher-lying Fermi level implies a weaker adsorption energy, since the electron is deposited at a higher energy. This is why clusters just past a magic number, characterized by a higher Fermi level but an otherwise similar spectrum, adsorb $\mathrm{H}$ more weakly than clusters just before a magic number. We can also see how the induced DOS integrates to zero only because the Fermi level is located at a gap between electronic shells: Within each electronic subshell there are fluctuations in the induced DOS which correspond to slight movements of the electronic shells but without the introduction of any extra charge. These cause $\eta(\epsilon)$ to locally deviate from 0 . The adsorption energy therefore may not depend simply on the Fermi energy in general, but must do so at the magic numbers. These results are consistent with previous findings for very small clusters, that $\mathrm{H}$ atoms effectively contribute their electron to the lowest unoccupied molecular orbital (LUMO), behaving like an extra $\mathrm{Au}$ atom. ${ }^{52,53}$

\section{B. Adsorption of $\mathrm{O}$}

Since the Newns-Anderson model only takes a single state into account while $\mathrm{O}$ has three $p$ states, we assume that each of the states hybridizes independently and contributes to the adsorption energy as per Eq. (14). Thus, we consider one Hamiltonian of the form (5) for each $p$ state with varying $v_{a k}$ and $s_{a k}$.

The $2 p_{x}$ and $2 p_{y}$ states are close to degenerate and have almost identical chemisorption functions. Figure 16(a) shows the average $\Delta(\epsilon)$ and $\Lambda(\epsilon)$ from the $\mathrm{O} 2 p_{x}$ and $2 p_{y}$ states. In this case, the weaker splitting leads to greater smearing of the states close to the $d$ band and between the electronic shells. The higher-lying peaks in $\Delta(\epsilon)$ correspond to coupling with of the electronic shells. Figure 16(b) shows the total 


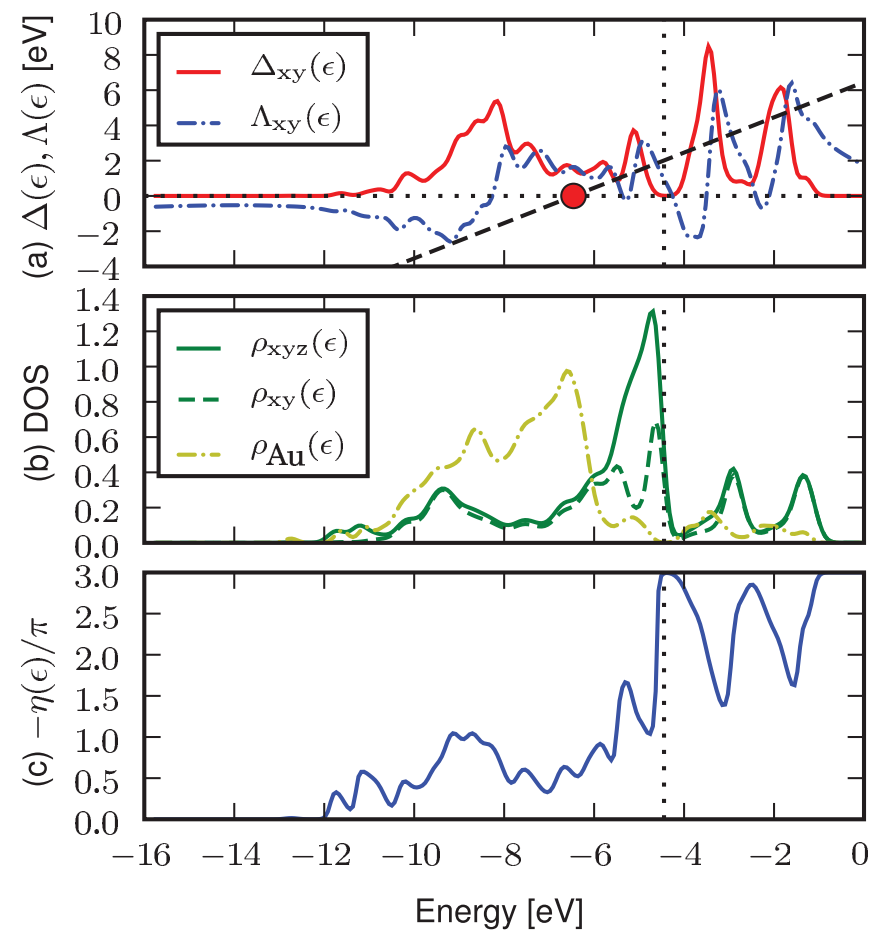

FIG. 16. (Color online) (a) $\Delta(\epsilon), \Lambda(\epsilon)$, and $\epsilon-\epsilon_{a}$ for $\mathrm{O} p_{x}$ or $p_{y}$ on a 58 -atom Au cluster. $\epsilon_{a}$ is indicated on the energy axis. (b) Projected DOS on all $\mathrm{O} 2 p$ states in $\mathrm{eV}^{-1}$ (solid line), on $p_{x}$ and $p_{y}$ only (dashed line), and total DOS of isolated cluster in arbitrary units. (c) Cumulative induced DOS.

PDOS $\rho_{x y z}(\epsilon)$ due to all three $p$ states (full lines) along with the contribution $\rho_{x y}(\epsilon)$ from $p_{x}$ and $p_{y}$ (dashed). The most profound feature is the state between the top of the $d$ band and the Fermi level, which therefore is filled. The induced DOS integrates to 3.0 at the Fermi level [Fig. 16(c)], allowing space for six electrons counting spin degeneracy. Since only four electrons are contributed, a total of two electrons are taken from the Fermi level into available lower-lying states. An upward shift in Fermi level therefore implies that more energy is gained from this transfer, causing a change in binding energy opposite that for $\mathrm{H}$ adsorption, as seen from the DFT calculations.

\section{Adsorption of $\mathrm{Li}$ and $\mathrm{F}$}

$\mathrm{Li}$ is the simplest of the four cases. Here the bonding is weak enough that no significant splitting occurs. The adsorbate state instead broadens into a resonance far above the Fermi level, see the left column of Fig. 17, without inducing any states below the Fermi level. The electron contributed by the $\mathrm{Li}$ atom therefore moves down to the Fermi level causing the same dependence of adsorption energy on Fermi level as for $\mathrm{H}$.

F couples more weakly than $\mathrm{O}$, and the bonding states are therefore split up less than for $O$ (right column of Fig. 17). Since both bonding and antibonding states are occupied, F behaves like $\mathrm{O}$ except only one electron can be transferred from the Fermi level, meaning that the change in adsorption energy at magic-number clusters is generally smaller than for $\mathrm{O}$.
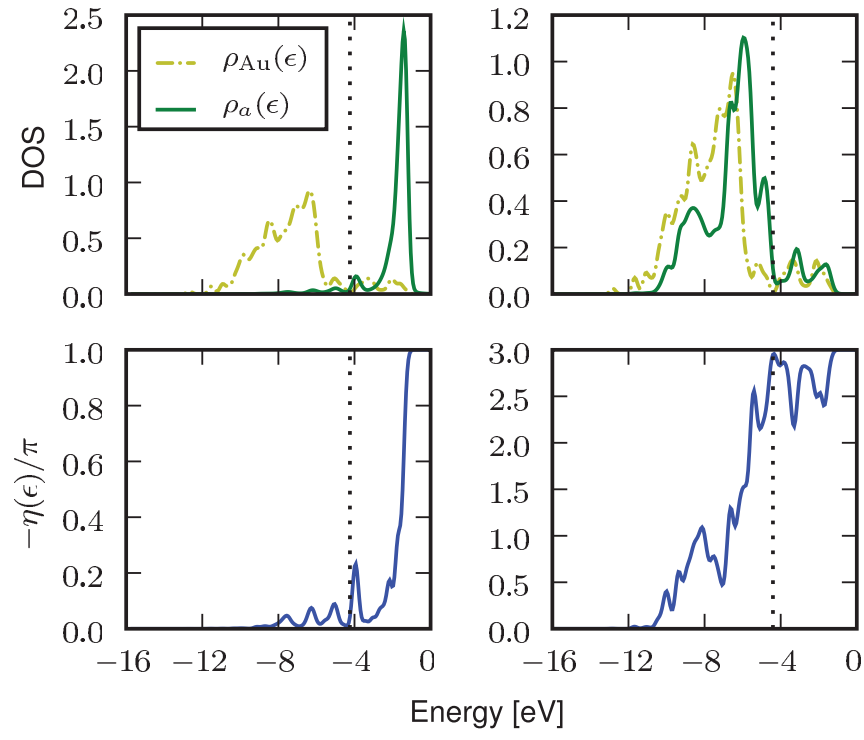

FIG. 17. (Color online) Projected density of states (top row) on adsorbate $\left(\mathrm{eV}^{-1}\right)$ and cluster (arbitrary units), and cumulative induced DOS (bottom row) for Li (left column) and F (right column).

\section{Comparison to Pt clusters}

Finally, we briefly consider the binding of $\mathrm{O}$ to the 58-atom Pt cluster. Again the $p_{x}$ and $p_{y}$ states are close to degenerate, and the average of their chemisorption functions is shown on Fig. 18(a). Two primary features appear in the chemisorption function: a strong coupling within the $d$ band around $\epsilon=$ $-8 \mathrm{eV}$ and a number of higher-lying peaks corresponding to electronic shells like those of Au clusters. Due to the broader and higher-lying $d$ band, the adsorbate state splits into peaks

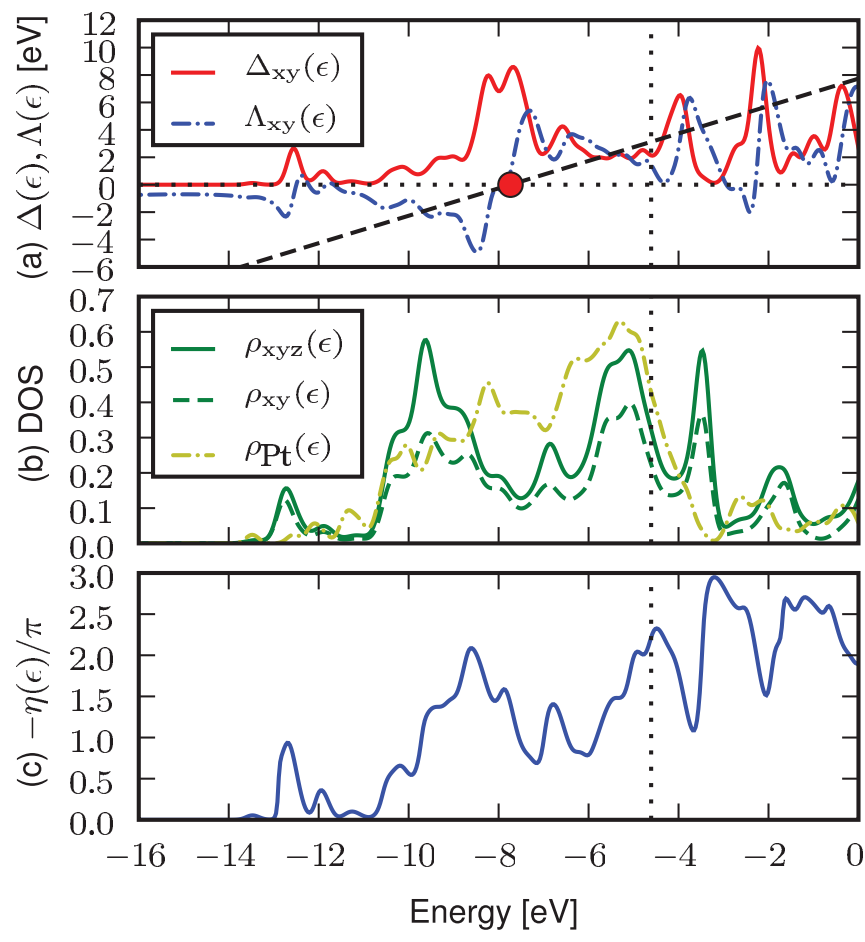

FIG. 18. (Color online) Chemisorption of O on 58-atom Pt cluster in comparison with Au from Fig. 16. 
over a wider energy range as seen on Fig. 18(b). The increase in binding on Pt compared to Au manifests itself as an increase in area below the curve in Fig. 16(c) [cf. Eq. (14)]. The overall upward shift of the coupling leads to an upward shift of the induced DOS, and so only approximately 2.2 of the 3 states contributed by $\mathrm{O}$ are in this case located below the Fermi level. The partial occupation of $\mathrm{O} 2 p$ states has been studied and confirmed experimentally. ${ }^{54}$ Recall that for Au, essentially all of the adsorbate-induced states were located below the Fermi level [Fig. 16(c)].

The $s$-electron shell structure is mostly visible in the chemisorption function well above the Fermi level. Further, since the location of the Fermi level within the $d$ band prevents abrupt changes in the Fermi level with cluster size, the $s$-electron shell structure-as expected-cannot exert a strong influence on the chemical binding on Pt clusters.

\section{CONCLUSIONS}

The structure of very small $\mathrm{Au}$ clusters is intricately dependent on the $s$-electron hybridization, with clusters at magic numbers having very large band gaps. Clusters with different numbers of atoms deform considerably to minimize the band structure energy by creating gaps at the Fermi level. For odd-numbered clusters this results in a singly filled state in the middle of the gap, causing strong even-odd oscillations of the highest occupied molecular orbital and LUMO.

Clusters based on regular geometries or a simple EMT potential show a more clear electronic shell structure and have large band gaps only at the major shell closings. These structures are less realistic, but are computationally feasible to optimize for larger cluster sizes.

Adsorption energies of atoms on regular $\mathrm{Au}$ clusters oscillate with the electronic magic numbers. While local geometry is known to be important, the variation in binding energy of $\mathrm{O}$ due to magic numbers alone may be up to $1 \mathrm{eV}$. Clusters just before or after magic numbers are found to exhibit roughly halogenlike and alkali-metal-like behavior while magic-number clusters are, as expected, universally unreactive.

A more detailed analysis attributes the increase or decrease in binding energy of specific adsorbates at magic numbers to properties of the adsorbate-induced DOS. Adsorption of $\mathrm{O}$ or $F$ induces states below the Fermi level, allowing the transfer of electrons from the Fermi level into the lower-lying states. In contrast, $\mathrm{H}$ and $\mathrm{Li}$, despite having very different adsorbate levels and electronegativity, only induce states above the Fermi level, and the electron contributed by these atoms is therefore transferred to the Fermi level.

\section{ACKNOWLEDGMENTS}

Center for Atomic-scale Materials Design is funded by the Lundbeck Foundation. The Catalysis for Sustainable Energy initiative is funded by the Danish Ministry of Science, Technology and Innovation. We acknowledge support from the Danish Center for Scientific Computing, from the Danish Council for Strategic Research's Programme Commission on Strategic Growth-Technologies (NABIIT), and from the U.S. Department of Energy-Basic Energy Sciences through the SUNCAT Center for Interface Science and Catalysis.
${ }^{1}$ M. Valden, X. Lai, and D. Goodman, Science (New York, NY) 281, 1647 (1998).

${ }^{2}$ H. A. Atwater and A. Polman, Nat. Mater. 9, 205 (2010).

${ }^{3}$ F. Baletto and R. Ferrando, Rev. Mod. Phys. 77, 371 (2005).

${ }^{4}$ D. G. Pettifor, J. Phys. F 8, 219 (1978).

${ }^{5}$ H. Häkkinen, B. Yoon, U. Landman, X. Li, H. Zhai, and L. Wang, J. Phys. Chem. A 107, 6168 (2003).

${ }^{6}$ E. M. Fernández, J. M. Soler, I. L. Garzón, and L. C. Balbás, Phys. Rev. B 70, 165403 (2004).

${ }^{7}$ X. Xing, B. Yoon, U. Landman, and J. H. Parks, Phys. Rev. B 74, 165423 (2006).

${ }^{8}$ H.-Y. Zhao, H. Ning, J. Wang, X.-J. Su, X.-G. Guo, and Y. Liu, Phys. Lett. A 374, 1033 (2010).

${ }^{9}$ W. D. Knight, K. Clemenger, W. A. de Heer, W. A. Saunders, M. Y. Chou, and M. L. Cohen, Phys. Rev. Lett. 52, 2141 (1984).

${ }^{10} \mathrm{G}$. Wrigge, M. Astruc Hoffmann, and B. v. Issendorff, Phys. Rev. A 65, 063201 (2002).

${ }^{11}$ T. P. Martin, Phys. Rep. 273, 199 (1996).

${ }^{12}$ A. S. Barnard, X. M. Lin, and L. A. Curtiss, J. Phys. Chem. B 109, 24465 (2005).

${ }^{13}$ A. S. Barnard, N. P. Young, A. I. Kirkland, M. A. van Huis, and H. Xu, ACS Nano 3, 1431 (2009).

${ }^{14}$ J. P. K. Doye and F. Calvo, Phys. Rev. Lett. 86, 3570 (2001).

${ }^{15}$ K. W. Jacobsen, J. K. Nørskov, and M. J. Puska, Phys. Rev. B 35, 7423 (1987)
${ }^{16}$ K. W. Jacobsen, P. Stoltze, and J. K. Nørskov, Surf. Sci. 366, 394 (1996).

${ }^{17}$ J. J. Mortensen, L. B. Hansen, and K. W. Jacobsen, Phys. Rev. B 71, 035109 (2005)

${ }^{18}$ J. Enkovaara, C. Rostgaard, J. J. Mortensen, J. Chen, M. Dułak, L. Ferrighi, J. Gavnholt, C. Glinsvad, V. Haikola, H. A. Hansen et al., J. Phys. Condens. Matter 22, 253202 (2010).

${ }^{19}$ B. Hammer, L. B. Hansen, and J. K. Nørskov, Phys. Rev. B 59, 7413 (1999).

${ }^{20}$ P. E. Blöchl, Phys. Rev. B 50, 17953 (1994).

${ }^{21}$ A. H. Larsen, M. Vanin, J. J. Mortensen, K. S. Thygesen, and K. W. Jacobsen, Phys. Rev. B 80, 195112 (2009).

${ }^{22}$ P. Pulay, Chem. Phys. Lett. 73, 393 (1980).

${ }^{23}$ S. R. Bahn and K. W. Jacobsen, Comput. Sci. Eng. 4, 56 (2002).

${ }^{24}$ L. Xiao, B. Tollberg, X. Hu, and L. Wang, J. Chem. Phys. 124, 114309 (2006).

${ }^{25}$ B. Yoon, P. Koskinen, B. Huber, O. Kostko, B. von Issendorff, H. Häkkinen, M. Moseler, and U. Landman, Chem. Phys. Chem. 8, 157 (2007).

${ }^{26}$ A. W. Snow and H. Wohltjen, Chem. Mater. 10, 947 (1998).

${ }^{27}$ J. Kleis, J. Greeley, N. A. Romero, V. A. Morozov, H. Falsig, A. H. Larsen, J. Lu, J. J. Mortensen, M. Dułak, K. S. Thygesen et al., Catal. Lett. 141, 1067 (2011).

${ }^{28}$ L. Ferrighi, B. Hammer, and G. K. H. Madsen, J. Am. Chem. Soc. 131, 10605 (2009) 
${ }^{29}$ E. M. Fernández, J. M. Soler, and L. C. Balbás, Phys. Rev. B 73, 235433 (2006).

${ }^{30}$ L. Xiao and L. Wang, Chem. Phys. Lett. 392, 452 (2004).

${ }^{31}$ ASAP, https://wiki.fysik.dtu.dk/asap.

${ }^{32}$ W. A. de Heer, W. Knight, M. Chou, M. L. Cohen, H. Ehrenreich, and D. Turnbull, in Advances in Research and Applications (Academic Press, San Diego, 1987), Vol. 40, pp. 93-181.

${ }^{33}$ M. Brack, Rev. Mod. Phys. 65, 677 (1993).

${ }^{34}$ O. Genzken and M. Brack, Phys. Rev. Lett. 67, 3286 (1991).

${ }^{35}$ I. Katakuse, T. Ichihara, Y. Fujita, T. Matsuo, T. Sakurai, and H. Matsuda, Int. J. Mass Spectrom. Ion Processes 67, 229 (1985).

${ }^{36}$ J. Li, X. Li, H. Zhai, and L. Wang, Science 299, 864 (2003).

${ }^{37}$ O. B. Christensen, K. W. Jacobsen, J. K. Nørskov, and M. Manninen, Phys. Rev. Lett. 66, 2219 (1991).

${ }^{38}$ O. B. Christensen and K. W. Jacobsen, J. Phys. Condens. Matter 5, 5591 (1993).

${ }^{39}$ Z. Penzar and W. Ekardt, Z. Phys. D 17, 69 (1990).

${ }^{40}$ M. Koskinen, P. O. Lipas, and M. Manninen, Z. Phys. D 35, 285 (1995).

${ }^{41}$ M. Moseler, H. Häkkinen, and B. Issendorff, in High Performance Computing in Science and Engineering '04, edited by E. Krause, W. Jäger, and M. Resch (Springer Berlin Heidelberg, Berlin, Heidelberg, 2005), pp. 95-105.
${ }^{42}$ P. Pyykkö and J. P. Desclaux, Acc. Chem. Res. 12, 276 (1979).

${ }^{43}$ H. Häkkinen, M. Moseler, and U. Landman, Phys. Rev. Lett. 89, 033401 (2002).

${ }^{44}$ J. Mansikka-aho, E. Hammarén, and M. Manninen, Phys. Rev. B 46, 12649 (1992).

${ }^{45}$ N. Lopez and J. K. Nørskov, J. Am. Chem. Soc. 124, 11262 (2002).

${ }^{46}$ H. Falsig, B. Hvolbæk, I. S. Kristensen, T. Jiang, T. Bligaard, C. H. Christensen, and J. K. Nørskov, Angew. Chem. 120, 4913 (2008).

${ }^{47}$ B. Hammer and J. K. Nørskov, Nature (London) 376, 238 (1995).

${ }^{48}$ J. K. Nørskov, A. Houmøller, P. K. Johansson, and B. I. Lundqvist, Phys. Rev. Lett. 46, 257 (1981).

${ }^{49}$ D. M. Newns, Phys. Rev. 178, 1123 (1969).

${ }^{50}$ A. Mackintosh and O. Andersen, Electrons at the Fermi Surface, edited by M. Springford (Cambridge University Press, London, 1980), p. 149.

${ }^{51}$ T. B. Grimley, J. Phys. C 3, 1934 (1970).

${ }^{52}$ S. Buckart, G. Ganteför, Y. D. Kim, and P. Jena, J. Am. Chem. Soc. 125, 14205 (2003).

${ }^{53}$ N. S. Phala, G. Klatt, and E. van Steen, Chem. Phys. Lett. 395, 33 (2004).

${ }^{54}$ P. Strasser, S. Koh, T. Anniyev, J. Greeley, K. More, C. Yu, Z. Liu, S. Kaya, D. Nordlund, H. Ogasawara et al., Nat. Chem. 2, 454 (2010). 